\title{
Origin of the vortex displacement field in twisted bilayer graphene
}

\author{
Yu. N. Gornostyrev ${ }^{1,2}$ and M. I. Katsnelson ${ }^{3,2}$ \\ ${ }^{1}$ M. N. Mikheev Institute of Metal Physics UB RAS, \\ 620137, S. Kovalevskaya str. 18, Ekaterinburg, Russia \\ ${ }^{2}$ Ural Federal University, Mira str. 19, 620002 Ekaterinburg, Russia \\ ${ }^{3}$ Radboud University, Institute for Molecules and Materials, NL-6525 AJ Nijmegen, The Netherlands
}

\begin{abstract}
Model description of patterns of atomic displacements in twisted bilayer systems has been proposed. The model is based on the consideration of several dislocation ensembles, employing a language that is widely used for grain boundaries and film/substrate systems. We show that three ensembles of parallel screw dislocations are sufficient both to describe the rotation of the layers as a whole, and for the vortex-like displacements resulting from elastic relaxation. The results give a clear explanation of the observed features of the structural state such as vortices, accompanied by alternating stacking.
\end{abstract}

PACS numbers:

\section{INTRODUCTION}

Bilayer systems consisting of two layers of identical or different two-dimensional materials such as bilayer graphene $(G / G)$, bilayer hexagonal boron nitride $(\mathrm{BN} / \mathrm{BN})$, and bilayer graphene/hexagonal boron nitride $(\mathrm{G} / \mathrm{BN})$ are the subject of great interest now as the simplest examples of "Van der Waals heterostructures" (for review, see Refs. ${ }^{[12}$ ). Building bilayer devices involves mechanical processes such as rotation and translation of one layer with respect to the other. This has substantial influence on the performance and quality of such devices ${ }^{3 / 4}$. The rotation leads to structural moiré patterns which directly affect the electronic properties of bilayers 5:11]. A further growth of interest to the field was triggered by a recent discovery of superconductivity and metal-insulator transition in "magic angle" twisted bilayer graphene $e^{12 \mid 13}$. In this paper, we focus on the structural aspects of moiré patterns and consider bilayer G/G. We suggest a description of the Moiré patterns in terms of vortices and in terms of dislocations and establish a connection between these two languages.

In two-dimensional materials, such as monolayer graphene, the term "dislocation" is typically used to describe pointlike (0D) defects lying within the sheet, e.g., pentagon-heptagon or square-octagon pairs $\frac{14}{\text {; }}$, they are also used to describe grain boundaries as dislocation wall 15 17. Such defects are edge dislocations with line directions oriented normal to the sheet. Unlike the case of monolayers, in bilayers it is also possible to have onedimensional (line) dislocations that lie between the two layers of a bilayer material; these dislocations do not require the generation of any topological defects within each of two sheets.

The geometry of displacement fields in bilayer Van der Waals systems has been discussed repeatedly starting from the discovery of commensurate-incommensurate transition in $\mathrm{G} / \mathrm{BN}$ system $\underline{\underline{9}}$. The results of atomistic simulations $\frac{18}{20}$ show that the formation of the vortex lattice is rather typical for the picture of displacements of the relaxed twisted bilayer systems (both G/BN and $\mathrm{G} / \mathrm{G}$ ). On the other hand, the electron microscopy study ${ }^{21}$ reveals multiple stacking domains with solitonlike boundaries between them in slightly twisted bilayer graphene, the domain boundaries can be also described as one-dimensional Frenkel-Kontorova dislocations. Wherein, a topological defect where six domains meet can be considered as vortex in displacement field. A similar multiple stacking domain structure was recently discussed in Ref ${ }^{[22}$ in the framework of a model employing a network of partial dislocation. However, the relation between these two descriptions, in terms of vortices and in terms of dislocations, is currently unclear.

Here, based on the dislocation model, we propose a general description of the moiré patterns in twisted bilayer graphene it terms of twist grain boundaries in layered material. We show that both pictures (vortex network and dislocation arrays) are consistent and presented two possible ways for a qualitative description of such systems and physical interpretation of the computer simulation results.

\section{DISLOCATION MODEL OF A TWISTED BILAYER SYSTEM}

Moiré patterns are formed initially by a rigid twist of the upper layer with respect to the bottom layer; their geometry is determined by lattice type and the rotation angle ${ }^{23}$. If one allows atomic relaxation, that is, their shifts from these ideal geometric positions within the lattice of coincidence sites to minimize the total energy, the picture becomes more complicated and, according to simulations 19 , vortex displacement field arises. Notably, the vortices form a regular lattice and are separated by broad regions of almost zero displacements.

There are two canonical ways to describe conjugation in bilayer systems. The first one is the simplest picture of coincidence site lattice (CSL) ${ }^{24}$ where one lattice just rotates and puts onto the other one without atomic relaxation; it corresponds to the moiré description. In the second approach, a general description of twist boundaries in 
bilayer systems can be derived on the basis of dislocation models proposed earlier for three-dimensional materials and thin films 25 . A consideration of grain boundaries based on the concept of surface dislocations was given in the book ${ }^{26}$ where general relations between grain boundaries and geometry of dislocation arrays were discussed. In the context of graphene, this language was used in Refs 15 17.

\section{A. General geometric relationships}

At least, two arrays of parallel equidistant screw dislocations are necessary to ensure a given relative twist of two crystallites in their conjugation plane ${ }^{26}$. In this case, certain geometric relations must be fulfilled so that the total shear deformation in the plane of the boundary is zero. In particular, in the case of two arrays, it is necessary to require that the dislocation axes in these arrays were perpendicular to each other.

To present correctly the geometry of conjugation of two graphene layers (and the corresponding moiré pattern), two dislocation arrays are not enough. We consider more general case and represent the plastic distortion tensor $\beta_{i j}^{p}$ produced by one array of dislocations in the form $\beta_{i j}^{p}=n_{i} b_{j} / d$, where $\mathbf{n}$ is the normal to the dislocation line lying in the plane of the boundary, $\mathbf{b}$ is the Burgers vector of dislocation, $d$ is the distance between neighboring dislocations in array. The plastic deformation $\varepsilon_{i j}^{p}$ and rotation $\omega_{i j}^{p}$ are determined by the symmetric and antisymmetric parts of the tensor $\beta_{i j}^{p}$

$$
\varepsilon_{i j}^{p}=\frac{n_{i} b_{j}+n_{j} b_{i}}{2 d} \quad \omega_{i j}^{p}=\frac{n_{i} b_{j}-n_{j} b_{i}}{2 d}
$$
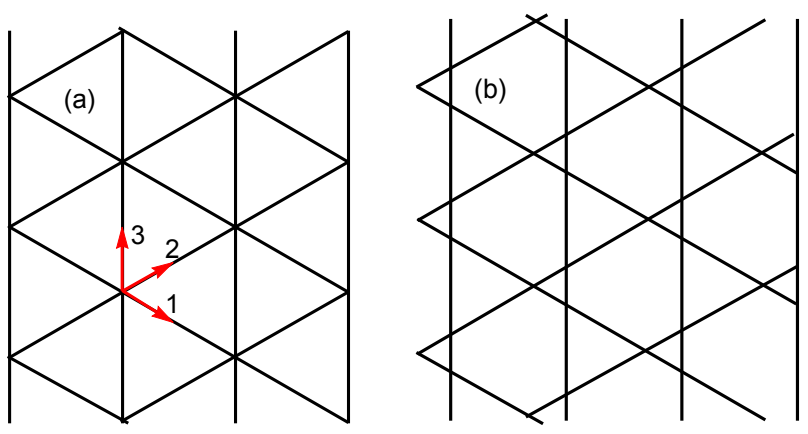

Figure 1: The schematic representation of the dislocation network used to describe the twist boundary. (a) - network of screw dislocations, (b) - reconstructed network of dislocations. Vectors 1,2,3 indicate the directions of dislocation lines.

To describe the conjugation of two twisted graphene layers and taking into account the lattice trigonal symmetry, we will use three dislocation arrays rotated with respect to each other by $\pi / 3$. Assuming that the normal to the graphene layers is [001] and considering screw dislocations with Burgers vector parallel to dislocation line, we write (see Fig. 1):

$$
\begin{array}{ll}
\mathbf{b}_{1}=b\left[\frac{\sqrt{3}}{2},-\frac{1}{2}, 0\right], & \mathbf{n}_{1}=\left[\frac{1}{2}, \frac{\sqrt{3}}{2}, 0\right], \\
\mathbf{b}_{2}=b\left[\frac{\sqrt{3}}{2}, \frac{1}{2}, 0\right], & \mathbf{n}_{2}=\left[-\frac{1}{2}, \frac{\sqrt{3}}{2}, 0\right]
\end{array}
$$

where $b$ is the module of the Burgers vector which should be equal to the elementary translation in graphene layer. To ensure the total deformation $\varepsilon_{i j}^{p}$ being equal to zero, it is necessary to choose the third dislocation array with the vector $\mathbf{b}_{3}$ orthogonal to $\mathbf{b}_{1}+\mathbf{b}_{2}$ :

$$
\mathbf{b}_{3}=b[0,1,0], \quad \mathbf{n}_{3}=[-1,0,0]
$$

Indeed, substituting expressions (2) and (3) into equation (1), we find

$$
\varepsilon_{i j}^{p}=0 \quad \omega_{i j}^{p}=\frac{3 b}{2 d}\left(\begin{array}{ccc}
0 & 1 & 0 \\
-1 & 0 & 0 \\
0 & 0 & 0
\end{array}\right)
$$

and the rotation vector $\omega_{k}=\frac{1}{2} \epsilon_{i j k} \omega_{i j}$ will be $\omega=$ $\frac{3 b}{2 d}[0,0,1]$. Note that for small rotation angle $\psi \approx \omega_{3}=$ $3 b /(2 d)$; this expression is similar to that determining the geometry of moiré in the model of CSL $\psi \sim a / l, l$ being the distance between coincidence points, $a$ being the elementary lattice translation.

\section{B. Relaxed displacement field within the dislocation model}

The equations (4) are valid on the average for the whole sheet. In fact, the displacements are non-uniformly distributed and concentrated near the dislocation lines. As discussed above, to describe correctly the displacement field in the case of twisted bilayer graphene three arrays of dislocations are necessary. We believe that the Frenkel-Kontorova mode $\frac{\sqrt{28}}{\text { gives a qualitatively correct }}$ description of the displacement field created by surface dislocations. Assuming that the energy relief of substrate may have an additional minimum $27 \mid 29$ and dislocations can split into the partial ones 2628 , the screw component of the displacement field for one family can be represented as

$$
\begin{aligned}
u_{s}(x)=\frac{b}{\pi} \sum_{i} & {\left[\arctan \left(\exp \left(\frac{x-x_{i}^{0}-\delta / 2}{\xi}\right)\right)\right.} \\
+ & \left.\arctan \left(\exp \left(\frac{x-x_{i}^{0}+\delta / 2}{\xi}\right)\right)\right]
\end{aligned}
$$

where $x_{i}^{0}$ correspond to position of the center of dislocation line, $\xi$ is core width and $\delta \sim \mu b / \gamma$ is distance splitting between partial dislocations, $\mu$ is shear modulus and $\gamma$ is stacking fault energy. 
The splitting of the dislocation on hexagonal lattice results in formation of stacking fault which is accompanied also by the appearance of an edge components of partial dislocations ${ }^{22}, \mathbf{b}=\left(\mathbf{b} / 2+\mathbf{b}_{e}\right)+\left(\mathbf{b} / 2-\mathbf{b}_{e}\right)$. In this case, the edge component of displacement field can be written as

$$
\begin{aligned}
u_{e}(x)=\frac{b_{e}}{\pi} \sum_{i} & {\left[\arctan \left(\exp \left(\frac{x-x_{i}^{0}-\delta / 2}{\xi}\right)\right)\right.} \\
- & \left.\arctan \left(\exp \left(\frac{x-x_{i}^{0}+\delta / 2}{\xi}\right)\right)\right]
\end{aligned}
$$

Fig. 22 displays the dependence $u_{s}(x)$ for the cases of narrow and wide (split) dislocations. In the case of narrow dislocations the displacements are concentrated in the dislocation core and include both plastic and elastic parts. When the width of the dislocation core $\xi$ increases, the dependence $u_{s}(x)$ becomes close to linear $u \approx u^{p}=b x / d$ and represents a pure plastic shear. Fig. $2 \mathrm{~b}$ shows the edge component of displacements in case of split dislocation.
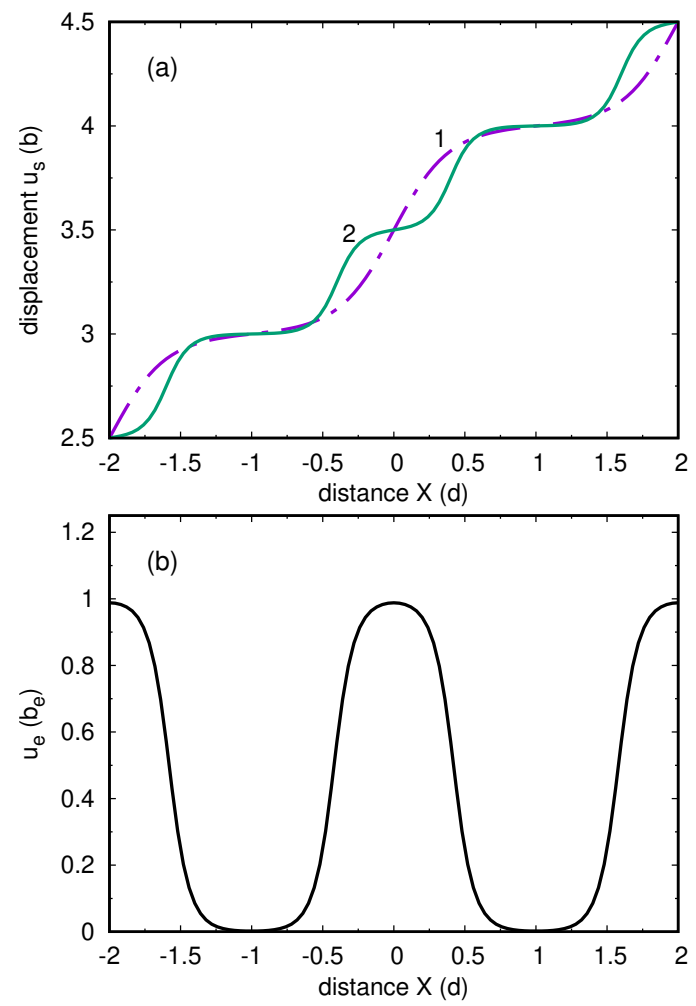

Figure 2: Screw component (a) of displacements produced by one dislocation array in the case of non-split $(\xi=0.2, \delta=0.2$, curve 1$)$ and split $(\xi=0.1, \delta=0.8$, curve 2$)$ dislocation cores. Edge component (b) of the displacements is produced by one array of partial dislocation $(\xi=0.1, \delta=0.8)$. Distances and parameters $\xi, \delta$ are given in units of $d$.

Following the discussion in the previous section, we represent the total displacement field in twisted graphene layers as a superposition of three dislocations arrays.
Subtracting the plastic part, we write the elastic displacements produced by screw dislocations in the form

$$
\begin{aligned}
\mathbf{u}^{e l}(\mathbf{r}) & =\sum_{k=1}^{3} \frac{\mathbf{b}_{k}}{\pi} \sum_{i=-m}^{m} \arctan \left(\exp \left(\frac{\mathbf{r n}_{k}-x_{i}^{k}-\delta / 2}{\xi}\right)\right) \\
& +\arctan \left(\exp \left(\frac{\mathbf{r n}_{k}-x_{i}^{k}+\delta / 2}{\xi}\right)\right) \\
& -\left(m b+\frac{b}{L} \mathbf{r n}_{k}\right)
\end{aligned}
$$

where $\mathbf{n}_{k}=[001] \times \mathbf{b}_{k}$ is the normal to the dislocation line of the k-th array. In addition, in the case of split dislocations, there is a displacement field created by arrays of edge partial dislocations

$$
\begin{aligned}
\mathbf{u}_{e}(\mathbf{r}) & =\sum_{k=1}^{3} \frac{\mathbf{b}_{k}^{p}}{\pi} \sum_{i=-m}^{m}\left[\arctan \left(\exp \left(\frac{\mathbf{r n}_{k}-x_{i}^{k}+\delta / 2}{\xi}\right)\right)\right. \\
& \left.-\arctan \left(\exp \left(\frac{\mathbf{r n}_{k}-x_{i}^{k}-\delta / 2}{\xi}\right)\right)\right]
\end{aligned}
$$

The vector fields described by equation $(7)$ are shown in Fig. 3 for the cases of narrow (non-split) and split dislocation cores. As one can see from Fig. 3 $3 a, b$, the screw component of the displacement field producing by elastic relaxation (7) forms a vortex lattice. However, the geometry of displacements is quite different for the cases under consideration. in particular, dislocation splitting results in a decrease of the period of vortex lattice in two times; the magnitude of displacement becomes essentially smaller (Fig. 3b). Distribution of the corresponding edge components of displacement field $\mathbf{u}_{\text {edge }}(\mathbf{r})$ is shown in Fig. 3p. Alternating domains of almost constant displacements correspond to different types of the stacking faults.

The picture of elastic displacement is rather similar to that obtained in atomistic simulations (see Ref's s9l29), $^{2}$ ), which indicates a semiquantitative correctness of the description of atomic relaxation effects in twisted graphene bilayers within our simple dislocation model.

It is worthwhile to note that in the center of vortex situated in the crossing of dislocation lines in Fig. 1 the relative displacement of the layers is equal to the half of elementary translation which results in formation of a stacking fault. To visualize this stacking fault, one needs to pass from elastic deformations $\mathbf{u}_{e l}(\mathbf{r})$ to the relative displacements between the layers described by equation (5)). In this case the more energetically favorable configuration is the one where one of the dislocation families is shifted from the symmetry position (Fig. 1b) by the value $\delta l$ in the direction normal to dislocation lines.

The distribution of the elastic displacements after such a reconstruction of the dislocation network is shown in Fig. 3 $\mathrm{d}$. One can see that the reconstruction of the dislocation network results in a drastic change of the displacement field $\mathbf{u}_{e l}(\mathbf{r})$ (c.f. Fig. 3 a and d). The regions with almost zero displacements are surrounded by six 

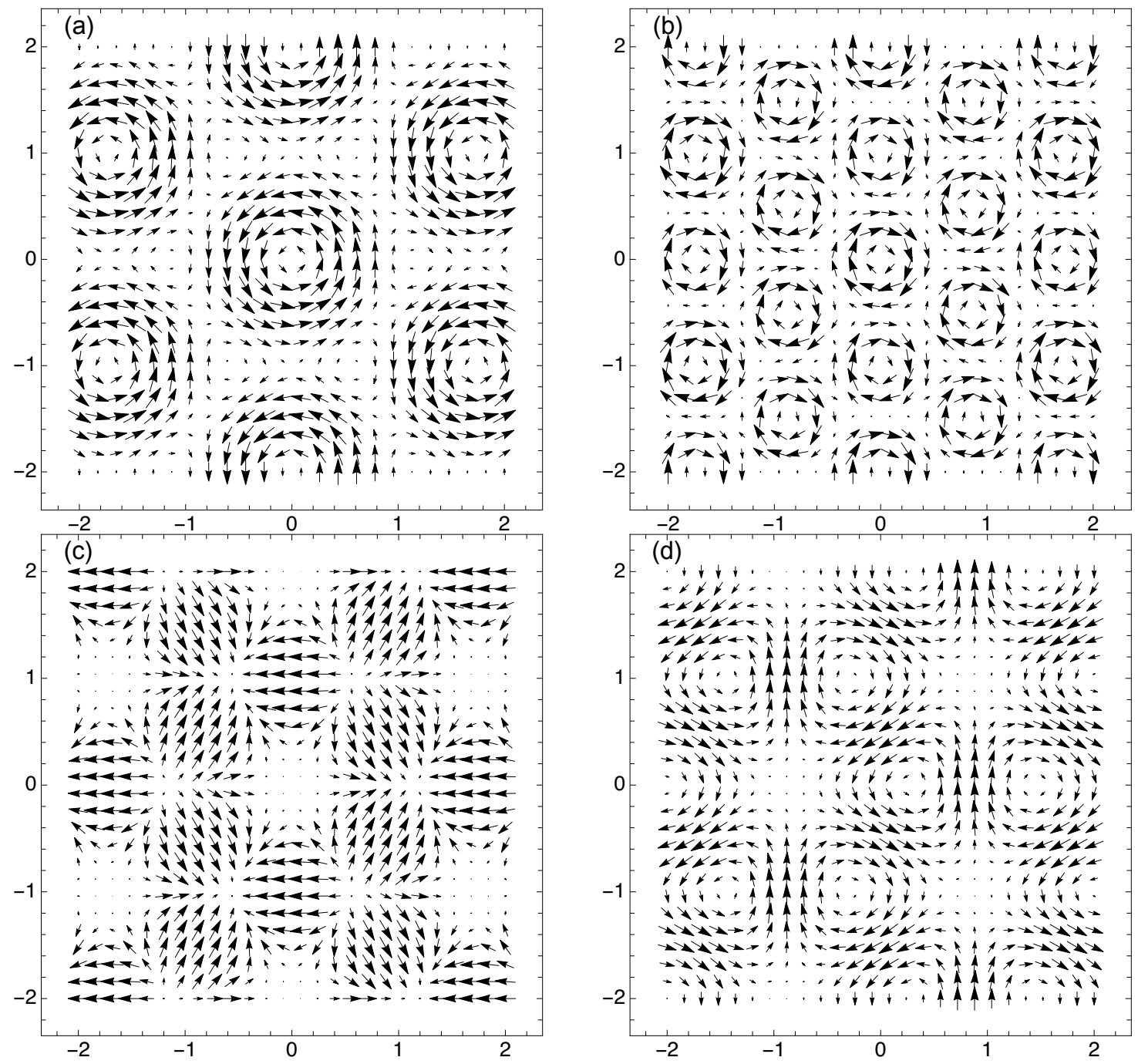

Figure 3: Displacement $\mathbf{u}_{e l}(\mathbf{r})$ shown as a vector field for narrow (a,d), split (b,c) dislocation cores $(\delta=0.4 d)$, and for the reconstructed dislocation network (d). (c) - edge component of displacement field $\mathbf{u}_{e}(\mathbf{r})$ in the case of split dislocation. $\xi$ is equal $0.05 d$ in cases $(\mathrm{a}, \mathrm{b}, \mathrm{c})$ and $\xi$ is equal $0.15 d$ in case $(\mathrm{d})$.

triangular regions with the largest displacements at their borders. According to Ref ${ }^{[22}$ the structure of conjugation of bilayer graphene can be described in terms of network of partial dislocations $\mathrm{a} / 3<1 \overline{100}>$ separating the regions of $\mathrm{AB}$ and $\mathrm{BA}$ stacking. The picture presented in Fig. $3 \mathrm{~d}$ agrees qualitatively with that discussed in Ref.22].

The other quantity characterizing peculiarities of the displacement field is the distribution of the rotation vector $\boldsymbol{\omega}(\mathbf{r})=\nabla \times \mathbf{u}_{\mathrm{el}}(\mathbf{r})$ presented in Fig. 4. As one can see from this figure, in the case of narrow core (Fig. 4 a), the dislocation lines are clearly visible in the distribution of the rotation vector and their intersections correspond to the centers of vortices. At the same time, the only lattice of vortices remains visible in the case of split dislocations. Thus, depending on the representation used, the conjugation of layers can be described either in terms of vortices or dislocations. Wherein, the vortex displace- ment field originates naturally from elastic relaxation of atomic positions. Note that the magnitude of rotation vector $\omega$ is close to zero for the edge component of displacements.

The other quantity which is actively discussed now $\sqrt{30}$ is the distribution of pseudomagnetic field $(\mathrm{PMF})^{2 / 31}$ given by the equations

$$
H_{P M F}=\frac{d A_{y}}{d x}-\frac{d A_{x}}{d y}
$$

where vector potential is expressed (with the accuracy of some constant multiplier) via deformations as

$$
A_{x}=u_{x x}-u_{y y}, \quad A_{x}=-2 u_{x y}
$$



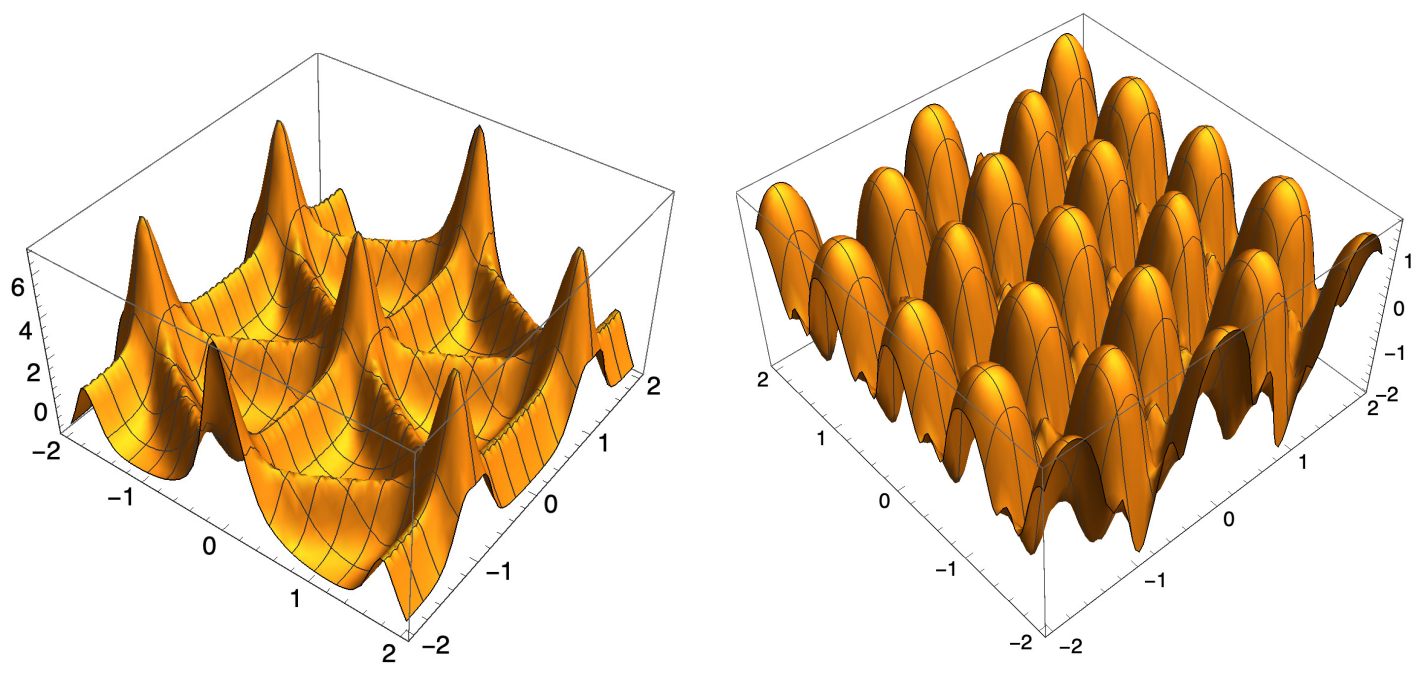

Figure 4: Distribution of z component of rotation vector $\boldsymbol{\omega}$ for narrow (left) and split (right) dislocation cores. The corresponding displacement fields are shown in Fig. 3 (a) and (b).

$$
\begin{array}{r}
u_{x x}=\frac{d u_{x}}{d x}+\frac{1}{2}\left(\frac{d u_{y}}{d x}\right)^{2}+\frac{1}{2}\left(\frac{d u_{y}}{d x}\right)^{2}, \\
u_{y y}=\frac{d u_{y}}{d y}+\frac{1}{2}\left(\frac{d u_{y}}{d y}\right)^{2}+\frac{1}{2}\left(\frac{d u_{x}}{d y}\right)^{2}, \\
u_{x y}=\frac{1}{2}\left(\frac{d u_{x}}{d y}+\frac{d u_{y}}{d x}+\frac{d u_{x}}{d y} \frac{d u_{x}}{d x}+\frac{d u_{y}}{d y} \frac{d u_{y}}{d x}\right)
\end{array}
$$

The distribution of PMF calculated from equations (9)-(10) for the reconstructed dislocation network with the displacement field from Fig. $3 \mathrm{~d}$ is shown in Fig. 5. Pink regions (with negative $\mathrm{PMF}$ ) correspond to the domains of small displacements in Fig. 3d; quasicircular yellow regions with positive PMF are situated in between. The presented picture is characteristic of the reconstructed dislocation network and will be much less regular for the other distributions of displacement fields considered here.

\section{DISCUSSION AND CONCLUSIONS}

The model developed here allows us to explain naturally some qualitative peculiarities of the moiré patterns in bilayer systems. In particular, the rotation angle and location of the coincidence site of the moire patterns are related to Burgers vector and the distance between dislocation lines (see Section IIa). We show that the plastic part of the displacement field provides rotation of one layer with respect to the other one as a whole. At the same time, the distribution of elastic displacements is quite complicated and vortices are the most typical element; the observed picture is in a qualitative agreement with both experiment $\underline{21}$ and the results of atomistic simulations 18,2022 .

Although, by construction, the displacements $\mathbf{u}^{\mathrm{el}}$ are

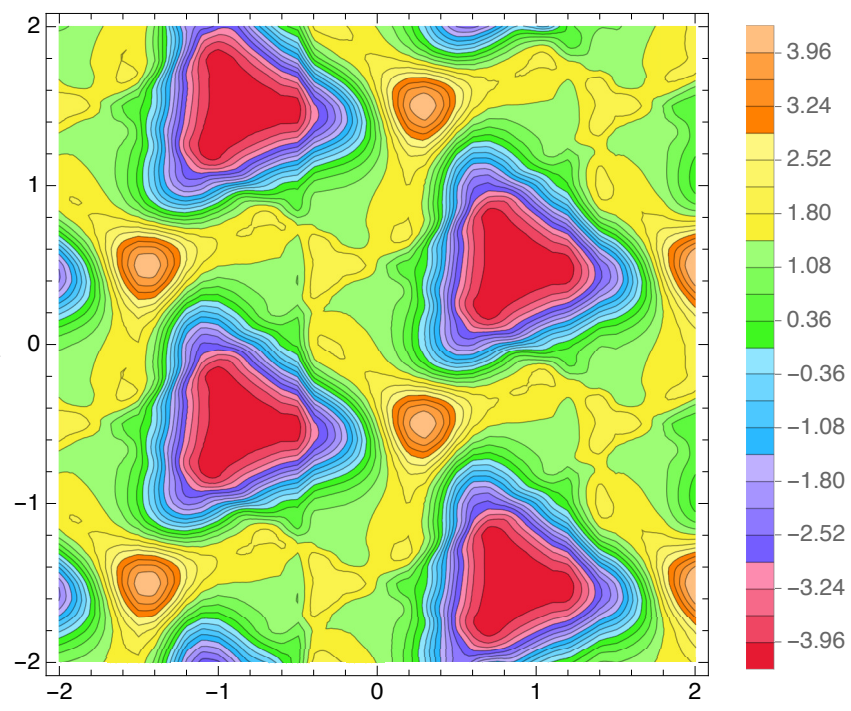

Figure 5: Distribution of PMF calculated for reconstructed dislocation network (Fig. 3d) by using Eq's (9)-(10)

equal to zero at a point located in the middle between two vortices, the derivative $d u_{i}^{e l} / d x_{j} \neq 0$. Note that the values $d u_{i}^{e l} / d x_{j}$ depend on distance between the dislocations in a given array and the width of their core. For narrow dislocations located far enough from each other $d u_{i}^{e l} / d x_{j} \approx 0$ in the middle between two vortices. However, a more realistic consideration of $\mathrm{G} / \mathrm{G}$ case ${ }^{22|27| 29}$ assumes the dislocation core splitting into partial dislocation cores. This means that the total dislocation core width is rather large, and the cores are situated rather close to each other. As a consequence, one should expect a remarkable deviation of the values $d u_{i}^{e l} / d x_{j}$ from zero between the vortices. By using 
equation (7) and assuming $L \gg z$ we have $d u_{x}^{e l} / d x_{y} \approx$ $2 b / \pi z \exp (-L / 2 z) \cosh (d / z)$. As a result, the region between the vortices is characterized by an excess of elastic energy density $\Delta E_{e l} \sim \mu\left(d u_{x}^{e l} / d x_{y}\right)^{2}$. This additional energy can be reduced by self-reorientation of the graphene layers ${ }^{20}$ resulting in the increase of the distance between dislocations (c.f. Eq. (4)).

Note, that although the model does not take into account a details related to the characteristics of chemical bonding and the formation of various types of stacking, it provides a clear vision of qualitative structural features of twisted bilayer systems. Our results unify the language used in the physics of moiré patterns in twisted bilayer graphene and other Van der Waals heterostructures with that traditionally used at the description of nano- and mesostructures in solids. We suggest explicit analytical expressions for the distribution of atomic displacements in twisted bilayer graphene which can be used for both model theoretical studies and interpretation of experimental and computational results.

\section{ACKNOWLEDGEMENTS}

This work of MIK was supported by the JTCFLAGERA Project GRANSPORT and work of YNG was financed by the Russian Ministry of education and science (topic "Structure" A18-118020190116-6).
1 A. K. Geim and I. V. Grigorieva, Nature 499, 419 (2013).

2 M. I. Katsnelson, The Physics of Graphene (2nd edition) (Cambridge Univ. Press, Cambridge, 2020).

3 G. Li, A. Luican, J. L. Dos Santos, A. Castro Neto, A. Reina, J. Kong, and E. Andrei, Nature Phys. 6, 109 (2009).

4 J. M. B. Lopes dos Santos, N. M. R. Peres, and A. H. Castro Neto, Phys. Rev. Lett. 99, 256802 (2007).

5 W.-T. Pong and C. Durkan, J. Phys. D 38, R329 (2005).

6 J. Xue, J. Sanchez-Yamagishi, D. Bulmash, P. Jacquod, A. Deshpande, K. Watanabe, T. Taniguchi, P. Jarillo-Herrero, and B. J. LeRoy, Nature Mater. 10, 282 (2011).

7 S. Tang, H. Wang, Y.u Zhang, A. Li, H. Xie, X. Liu, L. Liu, T. Li, F. Huang, X. Xie, and M. Jiang, Sci. Rep. 3, 2666 (2013).

8 W. Yang, G. Chen, Z. Shi, C.-C. Liu, L. Zhang, G. Xie, M. Cheng, D. Wang, R. Yang, D. Shi, K. Watanabe, T. Taniguchi, Y. Yao, Y. Zhang, and G. Zhang, Nature Mater. 12, 792 (2013).

${ }^{9}$ C. R. Woods, L. Britnell, A. Eckmann, R. S. Ma, J. C. Lu, H. M. Guo, X. Lin, G. L. Yu, Y. Cao, R. V. Gorbachev, A. V. Kretinin, J. Park, L. A. Ponomarenko, M. I. Katsnelson, Yu. N. Gornostyrev, K. Watanabe, T. Taniguchi, C. Casiraghi, H-J. Gao, A. K. Geim and K. S. Novoselov, Nature Phys. 10, 451 (2014).

10 G. J. Slotman, M. M. van Wijk, P.-L. Zhao, A. Fasolino, M.I. Katsnelson, and S. Yuan, Phys. Rev. Lett. 115, 186801 (2015).

${ }^{11}$ H. Shi, Z. Zhan, Z. Qi, K. Huang, E. van Veen, J. A. SilvaGuillen, R. Zhang, P. Li, K. Xie, H. Ji, M. I. Katsnelson, S. Yuan, S. Qin, and Z. Zhang, Nature Commun. 11, 371 (2020).

12 Y. Cao, V. Fatemi, S. Fang, K. Watanabe, T. Taniguchi, E. Kaxiras, and P. Jarillo-Herrero, Nature 556, 43 (2018).

13 Y. Cao, V. Fatemi, A. Demir, S. Fang, S. L. Tomarken, J. Y. Luo, J. D. Sanchez-Yamagishi, K. Watanabe, T. Taniguchi, E. Kaxiras, R. C. Ashoori, and P. JarilloHerrero, Nature 556, 80 (2018).

14 D. R. Nelson, Defects and Geometry in Condensed Matter Physics (Cambridge Univ. Press, Cambridge, 2002).

15 O. V. Yazyev and S. G. Louie, Nature Mater. 9, 806 (2010).
16 M. A. Akhukov, A. Fasolino, Y. N. Gornostyrev, and M. I. Katsnelson, Phys. Rev. B 85, 115407 (2012).

17 S. Dai, Y. Xiang, and D. J. Srolovitz, Phys. Rev. B 93, 085410 (2016).

18 M. M. van Wijk, A. Schuring, M. I. Katsnelson, and A. Fasolino, Phys. Rev. Lett. 113, 135504 (2014).

19 M. M. van Wijk, A. Schuring, M. I. Katsnelson, and A. Fasolino, 2D Mater. 2, 034010 (2015).

${ }^{20}$ C.R. Woods, F. Withers, M.J. Zhu, Y. Cao, G. Yu1, A. Kozikov, M. Ben Shalom, S.V. Morozov, M.M. van Wijk, A. Fasolino, M.I. Katsnelson, K. Watanabe, T. Taniguchi, A.K. Geim, A. Mishchenko, K.S. Novoselov, Nature Comm., 7, 10800 (2016).

21 J. S. Alden, A. W. Tsen, P. Y. Huang, R. Hovden, L. Brown, J. Park, D. A. Muller, and P. L. McEuen, Proc. Natl. Acad. Sci. USA 110, 11256 (2013).

22 S. Bagchi, H. T. Johnson, and H. B. Chew, Phys. Rev. B 101, 054109 (2020).

23 K. Hermann, J. Phys.: Cond. Mat. 24, 314210 (2012).

24 K. Sadananda and M.J. Marcinkowski, J. Appl. Phys. 45, 1521 (1974).

25 R. Siems, P. Delavignette, and S. Amelinckx, Phys. Status Solidi (b) 2, 421 (1962).

26 J. P. Hirth and J. Lothe, Theory of Dislocations (McGrawHill, New York, 1968).

27 S. Zhou, J. Han, S. Dai, J. Sun, and D. J. Srolovitz, Phys. Rev. B 92, 155438 (2015).

28 O. M. Braun and Y. S. Kishvar, The Frenkel-Kontorova Model: Concepts, Methods and Applications (Sptinger, Berlin, 2004).

${ }^{29}$ G. Savini, Y. J. Dappe, S. Öberg, J.-C. Charlier, M. I. Katsnelson, and A. Fasolino, Carbon 49, 62 (2011).

${ }^{30}$ H. Shi, Z. Zhan, Z. Qi, K. Huang, E. van Veen, J. A. SilvaGuillen, R. Zhang, P. Li, K. Xie, H. Ji, M. I. Katsnelson, S. Yuan, S. Qin, and Z. Zhang, Nature Commun. 11, 371 (2020).

31 M. A. H. Vozmediano, M. I. Katsnelson, and F. Guinea, Phys. Rep. 496, 109 (2010). 